\title{
Movilidad y reclutamiento de material leñoso de gran tamaño en dos cauces de la Cordillera de la Costa de Chile
}

\author{
In-stream large wood mobility and recruitment in two channels in the Coastal \\ Mountain Range, Chile
}

\author{
Andrés Iroumé ${ }^{*}$, Héctor Ulloa ${ }^{\text {b }}$, Mario A Lenzi c , Andrea Andreoli d, Carolina Gallo ${ }^{\mathrm{e}}$ \\ * Autor de correspondencia: ${ }^{\text {a } U n i v e r s i d a d ~ A u s t r a l ~ d e ~ C h i l e, ~ F a c u l t a d ~ d e ~ C i e n c i a s ~ F o r e s t a l e s ~ y ~ R e c u r s o s ~ N a t u r a l e s, ~ I n s t i t u t o ~ d e ~}$ \\ Manejo Forestal, Casilla 567, Valdivia, Chile, airoume@uach.cl \\ b Universidad Austral de Chile, Facultad de Ciencias Forestales y Recursos Naturales, Escuela de Graduados, Valdivia, Chile. \\ c Università degli studi di Padova, Facoltà di Agraria, Legnaro, Italia. \\ d Universidad de Concepción, Departamento de Manejo de Bosques y Medio Ambiente, Concepción, Chile. \\ e Universidad Politécnica de Madrid, Escuela de Ingeniería Forestal y del Medio Natural, Madrid, España.
}

\begin{abstract}
SUMMARY
In-stream large wood mobility was studied in segments of the main channels of Vuelta de Zorra and Pichún catchments, located in the Coastal Mountain Range, Chile. Statistical significant relationships were found only between bankfull channel width and dependent variables associated to some large wood characteristics measured in the channel reaches. This would indicate that large wood length, and not diameter, would regulate the mobility of the wood pieces during high flows. After the winter season of 2009 , the 12 and $6.5 \%$ of the wood elements were mobilized in Vuelta de Zorra and Pichún, respectively. The lower percentage in Pichún would be associated to the lower bankfull width/mean piece length ratio. A statistical non-significant trend was found between mobilized distance and piece length/mean bankfull width; indicating that distance decreased with increased dimensionless piece length. Large wood mobility in the two channels was associated to normal peak flows and could be considered as a minimum mobility rate at annual level. The recruited wood volume was higher than the exported wood volume in both segments, therefore the study period can be considered as an in-stream large wood stocking-period.
\end{abstract}

Key words: In-stream large wood, mobility, recruitment, mountain channels.

\section{RESUMEN}

Se estudió la movilidad de material leñoso de gran tamaño (LW) en segmentos de los cauces principales de las cuencas Vuelta de Zorra y Pichún, ubicadas en la Cordillera de la Costa, Chile. Se encontraron relaciones estadísticamente significativas entre el ancho del canal al nivel de cauce lleno (bankfull) de ambos cauces y variables dependientes asociadas a algunas características de las piezas de madera en cada tramo estudiado. Esto indicaría que la longitud de los elementos y no el diámetro regularía la movilidad del material durante crecidas. Luego de la temporada de invierno del año 2009, el 12 y el 6,5 \% del material leñoso se movilizó en las cuencas Vuelta de Zorra y Pichún. El menor porcentaje de piezas movilizadas en Pichún estaría dado por el menor cociente entre el ancho de bankfull y la longitud media de los LW. Se encontró una tendencia no significativa entre la distancia recorrida con la longitud no dimensional de las piezas de material leñoso (largo de la pieza/ancho medio de bankfull), que indicaría que la distancia recorrida disminuiría al aumentar la longitud adimensional de las piezas. La movilidad de LW en ambos cauces se asocia a la ocurrencia de crecidas normales, y podría considerarse una tasa mínima de movilidad a niveles anuales. Durante el estudio, se incorporó al cauce un volumen de madera mayor al que salió por transporte desde los segmentos estudiados, por lo que el periodo de estudio correspondió a uno de acumulación de material leñoso en ambos cauces.

Palabras clave: madera en el cauce, movilidad, reclutamiento, cauces de montaña.

\section{INTRODUCCIÓN}

Material leñoso de gran tamaño (large wood o LW en inglés) es todo trozo de madera de diámetro superior a $10 \mathrm{~cm}$ y largo mayor a 1 metro que se encuentra en los cauces y las llanuras inundables adyacentes (Fetherston et al. 1995). Estudios realizados en diferentes países y ambientes confirman la importancia que tiene la presencia de LW en la morfología, hidráulica y ecología de los ecosistemas fluviales. Una extensa descripción al respecto se encuentra en Ulloa (2010), Ulloa et al. (2011) y Wohl et al. (2010). Existe poca información en América Latina y menos en Chile sobre la abundancia y efectos morfológicos y ecológicos de la presencia de LW en ecosistemas fluviales. En el país, Andreoli et al. (2007), Mao et al. (2008) e Iroumé et al. (2010) informan respecto a la abundancia de LW y morfología asociada en cauces de la Cordillera de Los Andes y de la Costa, y Ulloa et al. (2011) comparan sobre la 
abundancia y distribución longitudinal de LW en cauces de dos cuencas de la Cordillera de La Costa con una historia contrastante del uso del territorio.

La cantidad y tipo de material leñoso existentes en los cauces dependen de las características del bosque de ribera (Comiti et al. 2006) y de los mecanismos que regulan el ingreso de LW a los mismos. Cuando la incorporación de LW se asocia sólo a la caída de árboles de la vegetación ribereña tanto por mortalidad natural (edad, viento) como por desestabilización por procesos erosivos de las orillas de los canales, la superficie que potencialmente puede aportar madera al cauce, por unidad de longitud del canal, es menor o igual a dos veces la altura del árbol más alto de esta vegetación (derivado de Robison y Beschta 1990). Deslizamientos de laderas o flujos torrenciales de canales afluentes pueden aportar LW al cauce principal desde terrenos alejados al mismo, extendiendo así el área potencial de suministro de madera. Por ejemplo, para el estero Tres Arroyos en Los Andes, Chile, Andreoli et al. (2007) reportan grandes acumulaciones de troncos en la confluencia de canales torrenciales de menor orden con el cauce principal.

La investigación sobre la abundancia y distribución longitudinal del material leñoso en cauces es en general abundante, pero la información sobre el inicio del transporte, movilidad y depósito de material leñoso es más escasa. Los LW se mueven durante crecidas importantes y varios autores presentan resultados sobre las características de distancia recorrida y de hidráulica de los trozos de madera que flotan en las aguas en estudios tanto en el campo como en experimentos de canales de prueba (Beebe 2000, Braudrick y Grant 2000, Bocchiola et al. 2002, Gurnell 2003). Los troncos pequeños tienen mayor movilidad que los grandes (Lienkaemper y Swanson 1987, Young 1994, Iroumé et al. 2010), la frecuencia de movimiento del material leñoso es mayor en cauces más anchos y profundos (Lienkaemper y Swanson 1987, Bilby y Ward 1989, Faustini y Jones 2003) y los elementos más móviles son los que tienen una longitud menor al ancho del canal en condiciones de cauce lleno o bankfull (Nakamura y Swanson 1994, Iroumé et al. 2010). Según Abbe y Montgomery (2003) los LW cuyo largo es mayor a 1,5 veces el ancho del canal de bankfull tienden a ser más estables, aun cuando Lienkaemper y Swanson (1987) reducen este umbral a la igualdad entre longitud del tronco y ancho de cauce lleno en el caso de corrientes de montaña de menor desarrollo. La permanencia de los LW en los canales depende también de la durabilidad natural la que varía en función de la especie (Juacida y Villanueva 1996) y de la presencia de trozos de madera "clave" de dimensiones mayores que anclan las acumulaciones de LW (o log jams) a las márgenes (Keller y Tally 1979, Bisson et al. 1987, Nakamura y Swanson 1994, Abbe y Montgomery 2003).

La hipótesis que se prueba es que la movilidad de los LW durante un periodo invernal está determinada por la cantidad de crecidas en las que se alcanza o supera el nivel de bankfull. Los objetivos de este trabajo son estudiar los patrones de distribución longitudinal de los LW y las características y condiciones que regulan la movilidad y reclutamiento de material leñoso en un segmento del cauce de dos cuencas en la Cordillera de la Costa, que se caracterizan por una contrastante historia de uso de suelo.

\section{MÉTODOS}

Área de estudio. El estudio se realizó en un segmento de los canales principales de los esteros Vuelta de Zorra y Pichún, respectivamente. En ambos segmentos, los canales están bien definidos y las condiciones de bankfull son consistentes.

La cuenca Vuelta de Zorra (39 58 $12^{\prime \prime}$ S) tiene una superficie de 586,8 ha y 431 ha la de Pichún (37030`12 $12^{\prime}$ $\mathrm{S})$, y ambas se localizan en la Cordillera de La Costa. La precipitación media anual en la estación Isla Teja de Valdivia (cercana a Vuelta de Zorra) y El Tambillo, comuna de Nacimiento (cercana a la cuenca Pichún), es 2.312 y $1.190 \mathrm{~mm}$, respectivamente. Mayores detalles sobre su localización, topografía, cubierta vegetal, suelos y geología están en Iroumé et al. (2010), Ulloa (2010) y Ulloa et al. (2011). Ambos cauces cuentan con estaciones fluviométricas ubicadas en secciones naturales y provistas de sensores de presión y sistemas de almacenamiento de datos que registran el nivel de aguas en forma continua.

La vegetación de ribera en la cuenca Vuelta de Zorra corresponde a un bosque siempreverde de 150 a 200 años de edad (Iroumé et al. 2010), con 2.040 árboles ha ${ }^{-1}$ y área basal de $63,1 \mathrm{~m}^{2}$ ha-1 que sube a $69,1 \mathrm{~m}^{2} \mathrm{ha}^{-1}$ al considerar los árboles muertos en pie (Ulloa et al. 2011). En Pichún, la vegetación de ribera tiene en promedio $25 \mathrm{~m}$ de ancho a cada lado del cauce, 662 árboles ha ${ }^{-1} \mathrm{y}$ un área basal de $23,1 \mathrm{~m}^{2}$ ha $^{-1}$ que sube a $24 \mathrm{~m}^{2}$ ha-1 si se consideran los muertos en pie. El 21 \% del área basal en Pichún corresponde a árboles de Pinus radiata (D. Don) remanentes de la plantación establecida el año 1975 y cosechada entre 2006 y 2007 (Ulloa et al. 2011).

Los mecanismos de incorporación de LW al cauce son similares en ambas cuencas, y estarían mayoritariamente asociados a la caída de árboles de la vegetación ribereña, por mortalidad natural o individuos que se desestabilizan por erosiones de las márgenes del canal. En ninguna de las cuencas se aprecia deslizamientos de laderas o flujos torrenciales de canales afluentes que pudieran aportar LW al cauce principal.

Caracterización de los segmentos de los cauces. Entre noviembre de 2008 y marzo de 2009 se estudió un segmento de $1.557 \mathrm{~m}$ de longitud del canal del estero Vuelta de Zorra y de $1.004 \mathrm{~m}$ de largo del canal principal del estero Pichún, en los que se midió y posicionó con respecto a puntos de control los LW y se caracterizó morfológicamente ambos cauces. Los segmentos estudiados fueron divididos en 16 tramos para Vuelta de Zorra y en 12 tramos para Pichún, definidos a base de la uniformidad de pendiente, sinuosi- 
dad, ancho del canal o abundancia de LW.

Usando un distanciómetro laser con inclinómetro se levantó el perfil longitudinal del talweg y definieron secciones transversales de cada tramo para determinar la pendiente del lecho y el ancho, profundidad media y máxima del canal correspondiente al nivel bankfull (Ulloa et al. 2011). Las características de los canales de los segmentos estudiados se presentan con detalle en Ulloa et al. (2011).

Todo trozo de madera de diámetro mayor a $10 \mathrm{~cm}$ y largo superior a $1 \mathrm{~m}$ ubicado en los cauces y orillas adyacentes se midió con huincha y forcípula para obtener su largo y diámetro medio y calcular el volumen. Cada LW se posicionó con respecto a elementos naturales (rocas, grandes árboles) y estacas de madera numeradas instaladas en los límites de cada tramo en ambos cauces. En gran parte de los LW se colocó una placa metálica numerada (los elementos de grandes dimensiones fueron marcados con hasta cinco placas) para estudiar la movilidad y distancia recorrida. Para cada pieza de LW se registró además el estado de degradación, tipo, especie, posición en el canal, orientación con respecto al flujo y origen (Ulloa et al. 2011). Usando como referencia las dimensiones del canal de bankfull (ancho medio, longitud y área) se determinó el volumen y abundancia unitarios de LW por cada tramo y cauce.

Los cauces fueron inspeccionados a mediados de diciembre de 2009 y principios del mes de enero de 2010 para Vuelta la Zorra y Pichún, respectivamente, para identificar los LW movilizados y los nuevos reclutamientos. Teniendo presente los datos medidos al inicio del estudio, se recorrió cada tramo de cauce para comprobar la localización de cada pieza, y en el caso de haberse desplazado, se registró su nueva posición. Los nuevos aportes de LW al cauce se registraron del mismo modo que se había hecho con los LW originales, midiendo sus dimensiones y localización en el canal, colocando en algunos de ellos una a varias chapas metálicas, posicionándolos respecto a algún punto de referencia y caracterizándolos.

Análisis estadísticos. Con Statgraphics ${ }^{\circledR}$ (versión 9.1) se desarrollaron regresiones simples para examinar los patrones de distribución longitudinal de los LW en el cauce, usando los parámetros del cauce (ancho medio y profundidad media máxima de bankfull y pendiente de cada tramo) como variables independientes y las características de los LW de cada tramo (volumen ha-1 ${ }^{-1}$ diámetro medio, largo medio, volumen medio por pieza, número de piezas $\mathrm{km}^{-1}$ y número de acumulaciones $\mathrm{km}^{-1}$ ) como variables dependientes, donde se toma el área o longitud del canal de bankfull como referencia.

En cuanto al recorrido de los elementos movilizados, se buscó relaciones entre las distancias recorridas por los LW con el largo no dimensional (largo del elemento/ancho medio de cauce lleno) y el diámetro no dimensional (diámetro del elemento/profundidad media de cauce lleno) de cada LW movilizado. Del mismo modo se analizó la relación entre distancia no dimensional recorrida por los elementos (distancia recorrida/diámetro del elemento movilizado) y el largo no dimensional de los elementos movilizados (longitud de la pieza/ancho de bankfull). Las regresiones se consideraron significativas cuando $P<0,1$.

\section{RESULTADOS}

Características de LW en los canales estudiados. Se encontró un total de 484 y 111 trozos de madera en Vuelta de Zorra y Pichún, de los cuales fueron individualizados mediante una o más chapas metálicas el $80 \%$ (388 piezas) y $70 \%$ (77 piezas), respectivamente. Tomando el área del canal bankfull como referencia, Ulloa et al. (2011) determinaron que el volumen medio de LW almacenado en los segmentos de Vuelta de Zorra y Pichún fue de 109,4 y $55,5 \mathrm{~m}^{3} \mathrm{ha}^{-1}$, respectivamente. Las dimensiones medias de los elementos de LW en el cauce fueron $24 \mathrm{~cm}$ de diámetro, $5,1 \mathrm{~m}$ de longitud y $0,4 \mathrm{~m}^{3}$ en volumen para Vuelta de Zorra y $22 \mathrm{~cm}$ de diámetro, $5,5 \mathrm{~m}$ de largo y un volumen de $0,2 \mathrm{~m}^{3}$ en Pichún. La distribución del tamaño de las piezas por rango de diámetro y largo está en Ulloa et al. (2011).

La distribución del volumen $\left(\mathrm{m}^{3} \mathrm{ha}^{-1}\right)$ y abundancia (piezas $\mathrm{km}^{-1}$ ) de LW mostró una gran variabilidad por tramos y segmentos de cauce, y aun cuando el tamaño medio por tramo de las piezas (diámetro y largo) resultó relativamente similar en ambos cauces, los mayores largos medios se registraron en Pichún y el mayor número de acumulaciones de LW $\left(\mathrm{N}^{\mathrm{o}} \mathrm{km}^{-1}\right)$ ocurrió en Vuelta de Zorra (cuadro 1).

Relaciones entre características de los canales y de los $L W$ en los segmentos estudiados. Entre las características del canal como variables independientes, se encontró relaciones estadísticamente significativas sólo entre el ancho de bankfull y variables dependientes asociadas a algunas características de los LW (cuadro 2).

En Vuelta de Zorra y Pichún se encontró una tendencia, estadísticamente significativa mostrando que la abundancia $\left(\right.$ piezas $\mathrm{km}^{-1}$ ) y la longitud (m) de las piezas de LW aumentaban al aumentar el ancho del canal de bankfull (figura 1). La relación entre la longitud de las piezas y el ancho del canal de bankfull muestra una tendencia asintótica a valores de longitud máximos (figura 1).

Movilidad y reclutamiento de $L W$. En el estero Vuelta de Zorra, de los 391 troncos marcados un total de 48 (12,3\%) no se encontraban en la posición registrada al inicio del estudio. Los elementos transportados tenían un diámetro entre 11 y $47 \mathrm{~cm}$, pero el $75 \%$ de ellos correspondía a las primeras dos clases de diámetro (se usan las mismas clases de diámetro y longitud de las piezas de LW definidas por Ulloa et al. (2011), para clasificar las dimensiones del material leñoso en estos cauces), es decir, tenían un diámetro inferior a $30 \mathrm{~cm}$ (figura 2). Respecto a la longitud, los elementos movilizados midieron entre 1,3 y $13 \mathrm{~m}$ y el $79 \%$ de ellos pertenecían a las dos primeras clases de longitud (menor a $7 \mathrm{~m}$ ) (figura 2). 
Cuadro 1. Características de los LW en los tramos en cada segmento de cauce estudiado. LW characteristics in every reach of the study segments.

\begin{tabular}{|c|c|c|c|c|c|c|c|c|c|c|c|c|}
\hline \multirow{2}{*}{$\begin{array}{l}\mathrm{N}^{\circ} \\
\text { Tramo }\end{array}$} & \multicolumn{2}{|c|}{$\begin{array}{l}\text { Volumen } \\
\left(\mathrm{m}^{3} \mathrm{ha}^{-1}\right)\end{array}$} & \multicolumn{2}{|c|}{$\begin{array}{l}\text { Diámetro medio } \\
\text { de los LW (cm) }\end{array}$} & \multicolumn{2}{|c|}{$\begin{array}{l}\text { Longitud media } \\
\text { de los LW (m) }\end{array}$} & \multicolumn{2}{|c|}{$\begin{array}{c}\text { Tamaño medio } \\
\text { pieza }\left(\mathrm{m}^{3}\right)\end{array}$} & \multicolumn{2}{|c|}{$\begin{array}{l}\text { Abundancia } \\
(\text { piezas km-1) }\end{array}$} & \multicolumn{2}{|c|}{$\begin{array}{l}\text { Acumulaciones } \\
\left(\mathrm{N}^{\mathrm{o}} \mathrm{km}^{-1}\right)\end{array}$} \\
\hline & VZ & $\mathrm{P}$ & $\mathrm{VZ}$ & $\mathrm{P}$ & VZ & $\mathrm{P}$ & $\mathrm{VZ}$ & $\mathrm{P}$ & $\mathrm{VZ}$ & $\mathrm{P}$ & $\mathrm{VZ}$ & $\mathrm{P}$ \\
\hline 1 & 124 & 63 & 23,9 & 19,9 & 5,0 & 3,7 & 0,4 & 0,1 & 255 & 230 & 11 & 7 \\
\hline 2 & 3 & 51 & 16,3 & 19,7 & 1,9 & 5,2 & 0,0 & 0,1 & 78 & 134 & 0 & 0 \\
\hline 3 & 50 & 133 & 28,2 & 22,5 & 4,0 & 6,2 & 0,3 & 0,4 & 155 & 135 & 0 & 7 \\
\hline 4 & 134 & 0 & 23,8 & 0,0 & 5,6 & 0,0 & 0,3 & 0,0 & 504 & 0 & 29 & 0 \\
\hline 5 & 33 & 45 & 17,6 & 19,7 & 5,0 & 6,6 & 0,1 & 0,2 & 311 & 146 & 17 & 16 \\
\hline 6 & 140 & 109 & 26,7 & 20,6 & 5,6 & 15,3 & 0,5 & 0,6 & 329 & 100 & 28 & 0 \\
\hline 7 & 245 & 9 & 25,3 & 18,0 & 5,6 & 3,6 & 0,5 & 0,1 & 648 & 46 & 27 & 0 \\
\hline 8 & 378 & 111 & 23,4 & 26,0 & 5,7 & 11,0 & 0,4 & 0,5 & 1.117 & 114 & 12 & 0 \\
\hline 9 & 217 & 51 & 28,5 & 28,5 & 5,5 & 4,4 & 0,7 & 0,5 & 433 & 12 & 29 & 0 \\
\hline 10 & 49 & 24 & 22,2 & 21,0 & 6,5 & 3,0 & 0,4 & 0,1 & 160 & 61 & 0 & 0 \\
\hline 11 & 34 & 57 & 24,3 & 24,0 & 4,7 & 3,3 & 0,3 & 0,2 & 118 & 109 & 0 & 7 \\
\hline 12 & 19 & 47 & 20,9 & 27,0 & 3,9 & 4,2 & 0,2 & 0,2 & 105 & 109 & 7 & 0 \\
\hline 13 & 54 & - & 22,6 & - & 3,6 & - & 0,2 & - & 227 & - & 16 & - \\
\hline 14 & 41 & - & 17,3 & - & 3,5 & - & 0,1 & - & 314 & - & 23 & - \\
\hline 15 & 52 & - & 29,1 & - & 3,5 & - & 0,3 & - & 166 & - & 10 & - \\
\hline 16 & 16 & - & 20,8 & - & 2,9 & - & 0,1 & . & 154 & - & 0 & - \\
\hline
\end{tabular}

VZ: Vuelta de Zorra. P: Pichún. LW: material leñoso de gran tamaño (large wood).

Cuadro 2. Relaciones estadísticas entre características del canal y características de los LW. Statistical relationships between channel and LW characteristics.

\begin{tabular}{|c|c|c|c|c|c|c|c|c|}
\hline \multirow{2}{*}{ 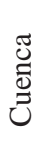 } & \multirow[b]{2}{*}{$\begin{array}{l}\text { Características del } \\
\text { canal }\end{array}$} & & \multicolumn{6}{|c|}{ Características de los LW } \\
\hline & & & $\begin{array}{l}\text { Volumen } \\
\left(\mathrm{m}^{3} \mathrm{ha}^{-1}\right)\end{array}$ & $\begin{array}{l}\text { Diámetro } \\
\quad(\mathrm{cm})\end{array}$ & $\begin{array}{l}\text { Longitud } \\
\text { (m) }\end{array}$ & $\begin{array}{c}\text { Tamaño medio } \\
\text { pieza }\left(\mathrm{m}^{3}\right)\end{array}$ & $\begin{array}{l}\text { Abundancia } \\
(\text { piezas km-1) }\end{array}$ & $\begin{array}{c}\text { Acumulaciones } \\
\qquad\left(\mathrm{N}^{\mathrm{o}} \mathrm{km}^{-1}\right)\end{array}$ \\
\hline \multirow{6}{*}{ 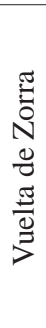 } & \multirow{2}{*}{ Ancho bankfull } & $\mathrm{R}^{2}$ & 0,26 & 0,05 & 0,49 & 0,31 & 0,31 & 0,18 \\
\hline & & $P$ & 0,04 & 0,40 & 0,00 & 0,02 & 0,03 & 0,10 \\
\hline & \multirow{2}{*}{ Profundidad máxima } & $\mathrm{R}^{2}$ & 0,15 & 0,05 & 0,04 & 0,15 & 0,07 & 0,00 \\
\hline & & $P$ & 0,14 & 0,43 & 0,46 & 0,13 & 0,32 & 0,83 \\
\hline & \multirow{2}{*}{ Pendiente } & $\mathrm{R}^{2}$ & 0,06 & 0,00 & 0,11 & 0,09 & 0,04 & 0,02 \\
\hline & & $\mathrm{P}$ & 0,38 & 0,98 & 0,21 & 0,27 & 0,48 & 0,65 \\
\hline \multirow{6}{*}{ 囬 } & \multirow{2}{*}{ Ancho bankfull } & $\mathrm{R}^{2}$ & 0,02 & 0,00 & 0,26 & 0,33 & 0,25 & 0,11 \\
\hline & & $P$ & 0,66 & 0,93 & 0,09 & 0,57 & 0,09 & 0,28 \\
\hline & \multirow{2}{*}{ Profundidad máxima } & $\mathrm{R}^{2}$ & 0,00 & 0,00 & 0,04 & 0,04 & 0,06 & 0,06 \\
\hline & & $P$ & 0,95 & 0,98 & 0,52 & 0,55 & 0,44 & 0,45 \\
\hline & \multirow{2}{*}{ Pendiente } & $\mathrm{R}^{2}$ & 0,00 & 0,14 & 0,04 & 0,01 & 0,00 & 0,00 \\
\hline & & $P$ & 0,88 & 0,24 & 0,54 & 0,75 & 0,99 & 0,93 \\
\hline
\end{tabular}

LW: material leñoso de gran tamaño (large wood). 

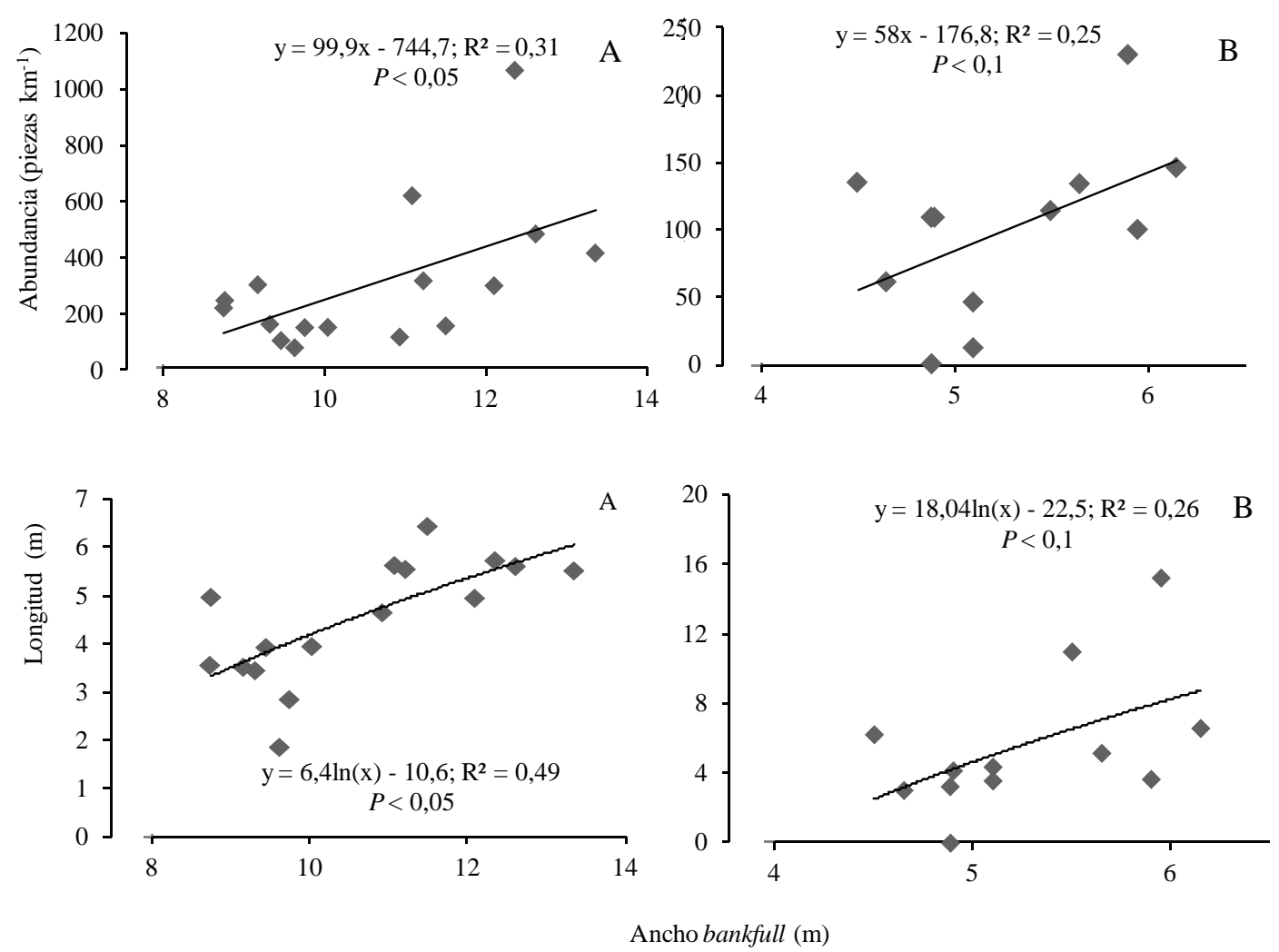

Figura 1. Relaciones entre el ancho medio de bankfull en cada tramo con la abundancia de LW (arriba) y la longitud media de las piezas (abajo). (A) Vuelta de Zorra, (B) Pichún.

Relationships between mean bankfull width and LW abundance (above) and mean length (below). (A) Vuelta de Zorra, (B) Pichún.
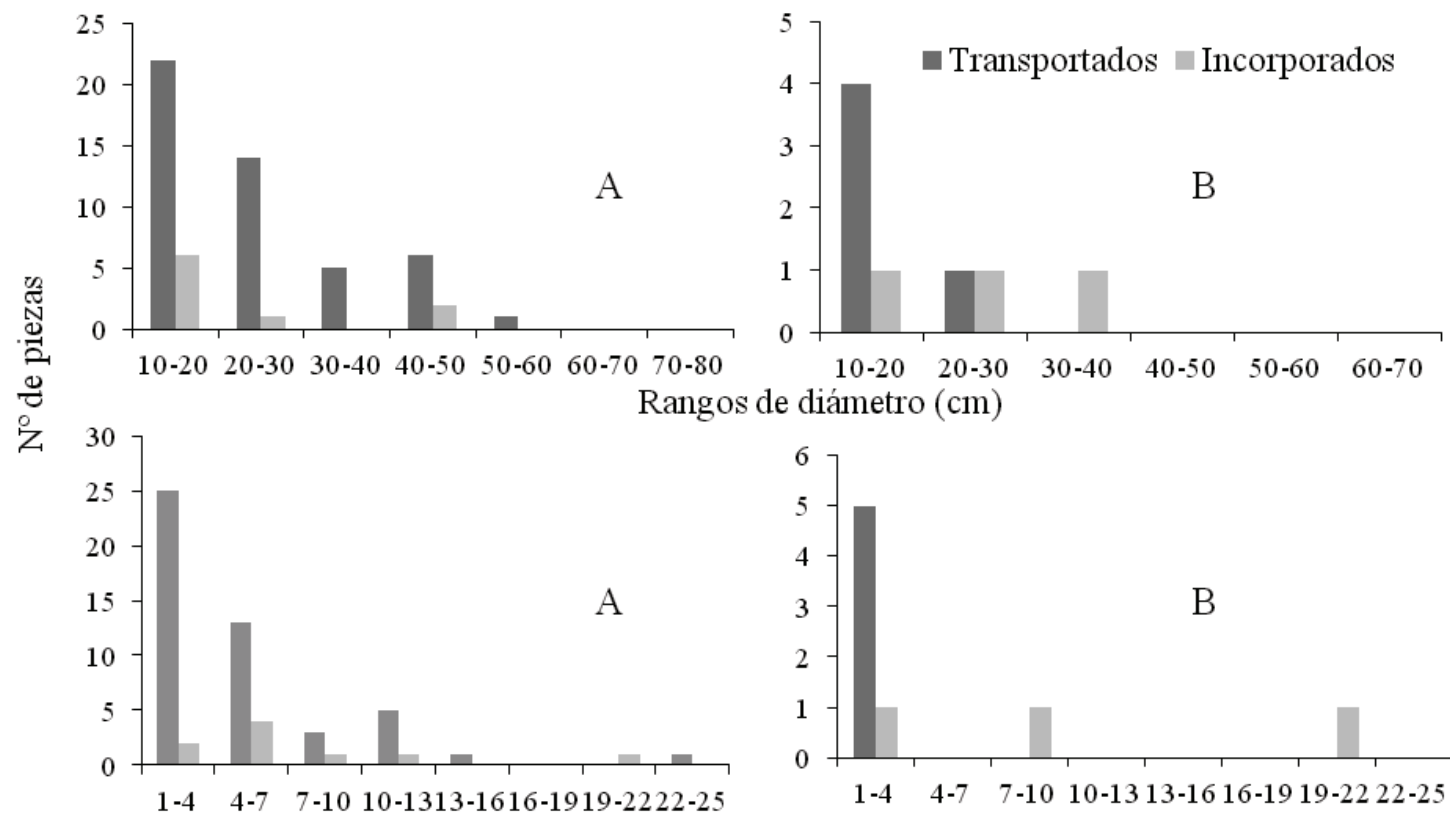

Rangos de longitud ( $\mathrm{m}$ )

Figura 2. Distribución del número de piezas de LW en rangos de diámetro (arriba) y de longitud (abajo) de los elementos movilizados e incorporados. (A) Vuelta de Zorra, (B) Pichún.

Distribution of number of LW pieces in diameter (above) and length ranges (below) of all mobilized and recruited wood elements. (A) Vuelta de Zorra, (B) Pichún. 
En Pichún, 5 de los 77 elementos marcados (es decir el 6,5\%) se habían movilizado. Los LW transportados tenían diámetros entre 12 y $26 \mathrm{~cm}$, y el $100 \%$ de las piezas se concentraba en las dos primeras clase de diámetros y el $80 \%$ en la primera clase (figura 2). Los elementos movilizados midieron entre 1,2 y $3,5 \mathrm{~m}$ correspondiendo el $100 \%$ a la primera clase de longitud (menor a $4 \mathrm{~m}$ ), figura 2 .

Respecto a las incorporaciones de LW al cauce, en Vuelta de Zorra se encontraron nueve elementos nuevos con diámetros entre 12 y $46 \mathrm{~cm}$, de los cuales el $67 \%$ eran menores a $20 \mathrm{~cm}$ (primera clase de diámetro), y sus longitudes se encontraban entre $1,2-20 \mathrm{~m}$, pero el $67 \%$ tenía menos de $7 \mathrm{~m}$ (primeras dos clases de longitud) (figura 2). En Pichún, se encontraron tres nuevos elementos con diámetros que fluctuaban entre 13,2 y $30 \mathrm{~cm}$ distribuidos en las primeras tres clases diamétricas, y con longitudes entre 3,7 y 19 m (figura 2). En ambos cauces, el reclutamiento fue predominantemente por mortalidad natural de árboles.

La movilidad de los LW se encuentra en general asociada a las mayores crecidas. Durante el periodo de estudio en ambas cuencas se observaron eventos donde la altura del agua alcanzó o superó por poco el nivel bankfull (figura 3), es decir, correspondieron a crecidas normales. La precipitación del año 2009 en Vuelta de Zorra y Pichún fue muy similar a la media anual de largo plazo en las zonas donde estas cuencas se localizan.

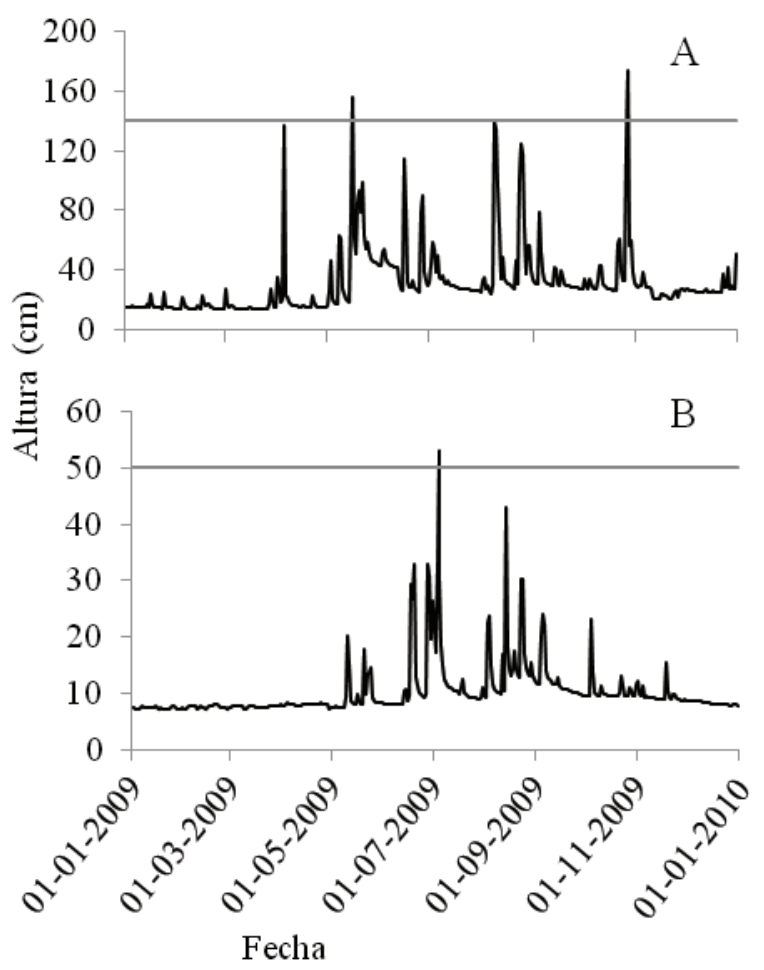

Figura 3. Alturas del agua en el cauce alcanzadas durante el período de estudio (línea horizontal indica el nivel de cauce lleno). (A) Vuelta de Zorra y (B) Pichún.

Stream water levels during the study period (horizontal line indicates bankfull stage), (A) Vuelta de Zorra and (B) Pichún.
Los elementos más cortos fueron movilizados a mayores distancias y se aprecia una tendencia (estadísticamente no significativa) entre la distancia recorrida con la longitud no dimensional de los LW (largo de la pieza/ancho medio de bankfull) (figura 4). De acuerdo a esta tendencia, la distancia recorrida disminuía al aumentar la longitud adimensional de las piezas de LW.

El volumen de LW incorporado al estero Vuelta de Zorra durante el periodo de estudio fue $4,3 \mathrm{~m}^{3}$ mientras que el volumen que salió de este segmento se estimó en $0,44 \mathrm{~m}^{3}$. Para Pichún el volumen incorporado al cauce fue $1,8 \mathrm{~m}^{3}$ y no se registró salida de elementos leñosos del segmento estudiado.

\section{DISCUSIÓN}

Características de los $L W$ en los segmentos estudiados. El diámetro es una de las dimensiones críticas de las piezas de madera porque condiciona la profundidad mínima del flujo requerida para iniciar el movimiento (Braudrick y Grant 2000). La longitud de los LW en relación con el ancho del canal también lo es, ya que los elementos más largos pueden quedar atrapados con otros árboles, atascados en el cauce o estabilizados en el margen del canal y la llanura de inundación reduciendo así el movimiento hacia aguas abajo (Gurnell et al. 2002). Iroumé et al. (2010) reportan que la longitud de los elementos es la variable que más influencia tiene en el desplazamiento de los LW durante crecidas.

El único parámetro del cauce que tiene relación estadísticamente significativa con los patrones de distribución longitudinal de los LW a lo largo del canal de ambos cauces estudiados es el ancho medio a nivel de cauce lleno (bankfull ) (cuadro 2 y figura 1). Esto también ocurre en el Whirinaki River en Nueva Zelanda, donde Baillie et al.

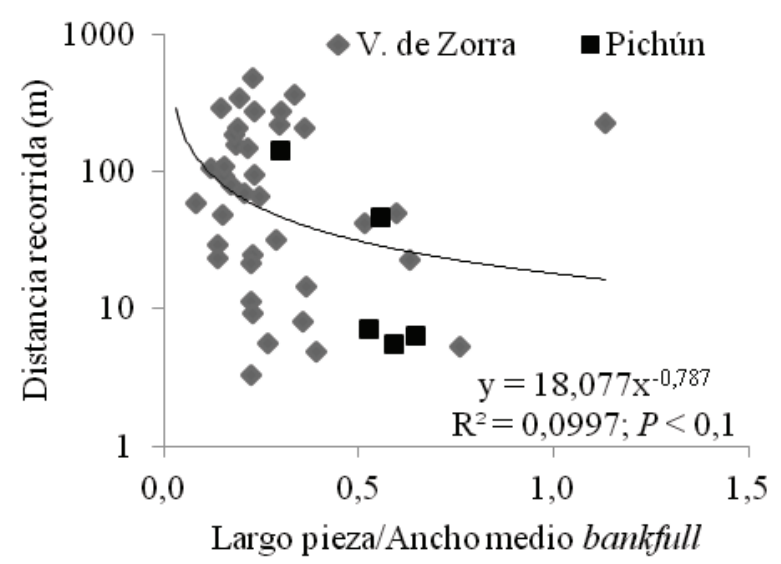

Figura 4. Relación entre la distancia de transporte de los troncos y la longitud adimensional de los troncos (largo de la pieza/ancho de canal de bankfull).

Relationship between LW transport distance and a dimensional piece length (piece length/bankfull width). 
(2008) encuentran que en la mayoría de los casos el ancho del canal correspondiente a cauce lleno presenta las correlaciones más altas con las características de los LW. En cuanto a las características de los LW, en ambos canales el diámetro no se correlaciona significativamente con ningún parámetro del cauce, lo cual puede deberse a que la profundidad al nivel de cauce lleno en los tramos de los segmentos estudiados es mayor al diámetro de todos los LW medidos.

Esto parece indicar que la longitud de los elementos y no el diámetro regularía la movilidad del material leñoso durante crecidas.

Movilidad y reclutamiento de $L W$. En Vuelta de Zorra se observan cifras de movilidad similares a lo que se encuentra en el torrente Buena Esperanza (Patagonia sur, Argentina), donde Mao et al. (2008) reportan el desplazamiento del $16 \%$ de los elementos de LW. En Pichún, el 6,5\% de los elementos de LW se movilizaron, valor cercano al $4 \%$ de movilidad en el Tres Arroyos que encuentran Andreoli et al. (2007). Valores mucho menores reportan Gurnell et al. (2002) que miden un movimiento de troncos de menos del $1 \%$ en el Mack Creek en Estados Unidos. Debe destacarse que tanto para Vuelta de Zorra como para Pichún, el periodo de estudio se caracteriza por la ocurrencia de crecidas normales, donde las aguas alcanzaron o superaron por muy poco el nivel del cauce lleno (figura 3). Sin embargo, en Vuelta de Zorra se registraron cuatro crecidas en las que el cauce alcanzó o superó por poco el nivel de bankfull, mientras que en Pichún sólo ocurrió una, cuestión que puede explicar la mayor movilidad en el primero de los cauces mencionados.

Las dimensiones de los troncos movilizados son comparables a las de los transportados en los torrentes Tres Arroyos (Cordillera de Los Andes, Chile) y Buena Esperanza (región argentina de Tierra de Fuego), con 15 $45 \mathrm{~cm}$ en diámetro y $1-5 \mathrm{~m}$ en longitud (Andreoli et al. 2007, Mao et al. 2008). El $97 \%$ y $100 \%$ de los elementos movilizados tiene una longitud menor al ancho medio del cauce para Vuelta de Zorra y Pichún, respectivamente, lo que coincide con otros estudios (Lienkaemper y Swanson 1987, Nakamura y Swanson 1994, Martin y Benda 2001) que concluyen que el largo de las piezas debe ser menor a la anchura del canal para que el transporte ocurra.

No se encuentra ninguna relación estadísticamente significativa entre la distancia recorrida por los LW movilizados y el diámetro de las piezas, lo que de alguna manera se puede asociar con los patrones de distribución de LW a lo largo de ambos cauces estudiados, donde se encuentra que el diámetro no tiene ninguna significación estadística al relacionarlo con los parámetros del cauce (cuadro 2). Para Vuelta de Zorra y Pichún se encuentra una tendencia (estadísticamente no significativa) entre distancia recorrida y longitud adimensional (largo de la pieza/ancho medio del canal de bankfull) de los LW movilizados (figura 4). En otros estudios, Andreoli et al. (2007) encuentran una correlación negativa entre la distancia de transporte y la longitud del tronco no dimensional en el torrente Tres Arroyos (Chile) y en cambio, en el caso del torrente Buena Esperanza (Argentina), Mao et al. (2008) reportan una alta dispersión de los valores sin una correlación estadísticamente significativa. Según lo reportado por Gurnell et al. (2002), ante avenidas ordinarias menos del $1 \%$ de los troncos parece estar sujeto a transporte y, frente a una avenida con recurrencia aproximada de 25 años, esta tasa apenas supera el $10 \%$ y de éstos, muy pocos son transportados una distancia de más de $10 \mathrm{~m}$. En Vuelta de Zorra y Pichún, el número de elementos movilizados fue mayor, posiblemente debido a un mayor número de avenidas ordinarias registradas durante el año de estudio. Martin y Benda (2001) encuentran que las distancias recorridas por los LW aumentan con el tamaño del canal, y que en los canales más pequeños hay un $90 \%$ de probabilidad de que los LW sean transportados al menos $50 \mathrm{~m}$ y un $10 \%$ de probabilidad de que el transporte exceda $300 \mathrm{~m}$, valores similares a los encontrados en el estero Vuelta de Zorra. La menor movilidad en el estero Pichún puede explicarse por las menores dimensiones del canal.

El valor de estos datos se destaca porque existe poca información de campo de transporte de material leñoso proveniente de investigaciones en el país. Esta información es de importancia para la evaluación del riesgo asociado al transporte de LW en cuencas de montaña, los que pueden causar daños a infraestructuras, y para el planteamiento de operaciones de mantención de la vegetación perifluvial en ríos urbanos en el caso de que este riesgo por movilidad de LW se considere importante.

El origen de los nuevos LW que llegan al cauce en Vuelta de Zorra y Pichún coincide con lo estudiado por Martin y Benda (2001), quienes determinan en el Game Creek (Alaska) que para cuencas menores de $10 \mathrm{~km}^{2}$ el reclutamiento es dominado por la mortalidad y en cuencas mayores por la erosión de las orillas. El suministro de elementos al cauce depende de la extensión y características de la vegetación de ribera, de ahí que la eliminación o reducción de grandes árboles en un bosque, originada por el uso histórico de los territorios, puede afectar significativamente al rol que juegan los LW en los procesos fluviales.

\section{CONCLUSIONES}

Ambos cauces presentaron desplazamiento de troncos. En Vuelta de Zorra la movilidad alcanzó a un $12 \%$ mientras en Pichún fue de un 6,5\% del total de los elementos marcados. El menor porcentaje de LW movilizados en Pichún estaría dado por el menor cociente entre el ancho de bankfull y la longitud media de los LW. Además se evidenció que el 97 y $100 \%$ de los LW movilizados no superó el ancho medio del cauce, en Vuelta de Zorra y Pichún, respectivamente, lo que concuerda con otros autores que determinan que el largo de las piezas movilizadas debe ser menor al ancho del canal para que el transporte ocurra.

La movilidad de LW en ambos cauces se asocia a la 
ocurrencia de crecidas normales, y una tasa cercana al $10 \%$ podría considerarse como tasa mínima de movilidad a niveles anuales. Esta información puede servir al diseño de obras hidráulicas en zonas de montaña en cauces que cruzan cuencas cubiertas con bosques.

El ingreso de nuevos elementos al cauce estuvo dominado por la caída de árboles y ramas de la vegetación de ribera, registrándose mayor aporte de madera en Vuelta de Zorra donde la vegetación de orilla de cauce es de mayores dimensiones. El mayor volumen de madera que se incorporó al cauce en relación al que salió por transporte desde los segmentos estudiados, indica que el periodo de estudio correspondió a uno de acumulación de LW en ambos cauces.

Estos resultados están basados en una investigación en dos cauces y durante sólo un periodo invernal. Esto ha permitido comprender parcialmente los procesos de movilidad y reclutamiento de LW específicos a ambos sitios pero no es posible aún generalizar para otros cauces y cuencas.

\section{AGRADECIMIENTOS}

Esta investigación se realizó en el marco del Proyecto FONDECYT 1080249. Los autores agradecen a The Nature Conservancy y Forestal Mininco S. A. por su interés y apoyo en los estudios que se están realizando en las cuencas Vuelta de Zorra y Pichún respectivamente. Los autores agradecen también las observaciones y sugerencias de dos árbitros anónimos que ayudaron a precisar aspectos del documento.

\section{REFERENCIAS}

Abbe T, D Montgomery. 2003. Patterns and processes of wood debris accumulation in the Queets river basin, Washington. Geomorphology 51: 81-107.

Andreoli A, G Carlig, F Comiti, A Iroumé. 2007. Residuos leñosos de gran tamaño en un torrente de la Cordillera de los Andes, Chile: su funcionalidad e importancia. Bosque 28(2): 83-96.

Baillie B, L Garrett, AEvanson. 2008. Spatial distribution and influence of large woody debris in an old-growth forest river system, New Zealand. Forest Ecology and Management 256: 20-27.

Beebe T. 2000. Flume studies of the effect of perpendicular log obstructions on flow patterns and bed topography. The Great Lakes Geographer 7(1): 9-25.

Bilby R, J Ward. 1989. Changes in characteristics and function of woody debris with increasing size of streams in western Washington. Transactions of the American Fisheries Society 118: 368-378.

Bisson P, R Bilby, M Bryant, C Doloff, G Grette, R House, M Murphy, K Koski, J Sedell. 1987. Large woody debris in forested streams in the Pacific Northwest: past, present and future. In Salo EO, TW Cundy eds. Streamside Management: Forestry and fishery interactions. Seattle, USA. College of Forest Resources, University of Washington. p. 143-190.

Bocchiola D, F Catalano, G Menduni, G Passoni. 2002. An analytical-numerical approach to the hydraulics of floating debris in river channels. Journal of Hydrology 269: 65-78.
Braudrick C, G Grant. 2000. When do logs move in rivers? Water Resources Research 36 (2): 571-583.

Comiti F, A Andreoli, MA Lenzi, L Mao. 2006. Spatial density and characteristics of woody debris in five mountain rivers of the Dolomites (Italian Alps). Geomorphology 78 (1-2): 44-63.

Faustini J, J Jones. 2003. Influence of large woody debris on channel morphology and dynamics in steep, boulder-rich mountain streams, western Cascades, Oregon. Geomorphology 51: 187-205.

Fetherston K, R Naiman, R Bilby. 1995. Large woody debris, physical process, and riparian forest development in montane river networks of the Pacific Northwest. Geomorphology 13: 133-144.

Gurnell A, H Piégay, F Swanson, S Gregory. 2002. Large wood and fluvial processes. Freshwater Biology 47: 601-619.

Gurnell A. 2003. Wood storage and mobility. In Gregory SV, KL Boyer, AM Gurnell eds. The ecology and management of wood in world rivers. Bethesda, Maryland, USA. American Fisheries Society. p. 75-91.

Iroumé A, A Andreoli, F Comiti, H Ulloa, A Huber. 2010. Large wood abundance, distribution and mobilization in a third order Coastal mountain range river system, southern Chile. Forest Ecology and Management 260: 480-490.

Juacida R, J Villanueva. 1996. Durabilidad natural de Sequoia sempervirens (D. Don) Endl. Bosque 17(2): 7-13.

Keller E, T Tally. 1979. Effects of large organic debris on channel form and fluvial processes in the coastal Redwood environment. In Rhodes DD, GP Williams eds. Adjustments of the fluvial system. Dubuque, IA, USA. Kendal-Hunt Publishing. p. 169-197.

Lienkaemper G, F Swanson. 1987. Dynamics of large woody debris in streams in old-growth Douglas-fir forests. Canadian Journal of Forest Research 17: 150-156.

Mao L, S Burns, F Comiti, A Andreoli, A Urciuolo, M GaviñoNovillo, R Iturraspe, MA Lenzi. 2008. Acumulaciones de detritos leñosos en un cauce de montaña de Tierra del Fuego: análisis de la movilidad y de los efectos hidromorfológicos. Bosque 29 (3): 197-211.

Martin D, L Benda. 2001. Patterns of instream wood recruitment and transport at the watershed scale. American Fisheries Society 130: 940-958.

Nakamura F, FJ Swanson. 1994. Distribution of coarse woody debris in a mountain stream, western Cascades Range, Oregon. Canadian Journal of Forest Research 24: 2395-2403.

Robison EG, RL Beschta. 1990. Identifying trees in riparian areas that can provide coarse woody debris to streams. Forest Science 36(3): 790-801.

Ulloa H, A Iroumé, MA Lenzi, A Andreoli. 2011. Material leñoso de gran tamaño en dos cuencas de la Cordillera de la Costa de Chile con diferente historia de uso del suelo. Bosque 32(3): 235-245.

Ulloa H. 2010. Material leñoso de gran tamaño en cauces de montaña. Tesis Magíster en Ciencias. Valdivia, Chile. Facultad de Ciencias Forestales y Recursos Naturales, Universidad Austral de Chile. 61 p.

Wohl E, DA Cenderelli, KA Dwire, SE Ryan-Burkett, MK Young, KD Fausch. 2010. Large in-stream wood studies: a call for common metrics. Earth Surface Processes and Landforms 35: 618-625.

Young M. 1994. Movement and characteristics of stream-borne coarse woody debris in adjacent burned and undisturbed watersheds in Wyoming. Canadian Journal of Forest Research 24: 1933-1938. 